\title{
Quantitative Analysis of the Elements of Interpersonal Trust of Poles
}

\author{
Doi:10.5901/mjss.2014.v5n13p72
}

\author{
Alicja Grześkowiak \\ Wrocław University of Economics \\ Email: alicja.grzeskowiak@ue.wroc.pl
}

\begin{abstract}
Interpersonal trust is a crucial element of the social trust and contributes essentially to economic growth. Various studies show that the level of interpersonal trust in Poland is relatively low what is an unfavorable situation. This paper aims at giving a statistical insight into interpersonal trust elements distinguished in the European Social Survey which can be described as trustfulness, helpfulness and fairness. These three components are considered with respect to some socio-economic characteristics as gender and age. As the dataset used in this study comprises both metric and non-metric variables several analytical methods are applied to explore the structure and nature of relationships. Additionally, the analyses are supported by appropriate data visualization techniques.
\end{abstract}

Keywords: interpersonal trust, statistical analysis

\section{Introduction}

Various studies indicate that interpersonal trust plays an important role in a societal and economic sense. An overview given by Morrone et al. (2009) describes possible positive relationships between interpersonal trust and many economic and social aspects. The lack of trust can contribute negatively to progress and development of the societies. In this context, the situation in Poland seems very unfavorable since the level of the interpersonal trust is relatively low what is proved by diverse surveys: World Value Survey (see Morrone et al. 2009, p.12), Social Diagnosis (see Czapiński \& Panek, 2013, p.286), Zaufanie społeczne (2012, p.2). Interpersonal trust is also an issue taken into account in the European Social Survey in which three elements of it may be distinguished: trustfulness, helpfulness and fairness. The relations among these three elements are considered by Grześkowiak (2014). This paper is an extension of previous work in which additional features are taken into consideration.

Main objective of this study is to analyze three components of interpersonal trust of Poles defined as: trustfulness, fairness and helpfulness in respect with such factors as gender and age. A general hypothesis which is put forward claims that the level of interpersonal trust may be affected by gender and age.

Hence, the main objective is accompanied by some specific objectives as:

- identification of the direction and strength of association between gender or age and interpersonal trust components,

- evaluation of the degree of influence of gender and age towards the perception of interpersonal trust.

\section{Data Source and Research Methods}

The analyses are based on data from the last wave of European Social Survey' (Wave 6 concerning year 2012), namely the answers to three questions reflecting components of interpersonal trust:

- "Would you say that most people can be trusted, or that you can't be too careful in dealing with people?"

- "Do you think that most people would try to take advantage of you if they got the chance, or would they try to be fair?"

- "Would you say that most of the time people try to be helpful or that they are mostly looking out for themselves?" 2

The respondents assessed their attitude on an eleven point Likert scale, for which extreme values were formulated in the survey as follows:

- „You can't be too careful” (0) to "Most people can be trusted” (10)

\footnotetext{
1 This paper uses European Social Survey data: ESS Round 6: European Social Survey Round 6 Data (2012). Data file edition 1.1, Norwegian Social Science Data Services, Norway - Data Archive and distributor of ESS data.

2 http://nesstar.ess.nsd.uib.no/webview/ [retrieved 2014-01-10]
} 
- "Most people try to take advantage of me" (0) to "Most people try to be fair" (10)

- "People mostly look out for themselves” (0) to „People mostly try to be helpful” (10) ${ }^{3}$

In this paper abbreviations for the three abovementioned questions are used, especially on figures, respectively: trustfulness, fairness, helpfulness.

The other data considered in this paper are two descriptive features, i.e. gender and age. It should be underlined that the analyses are carried out on the basis of mixed type data:

- nominal (gender),

- ordinal (attitudes towards interpersonal trust),

- ratio scale (age).

what requires particular procedures of the analysis. The analytical methods applied in this study include:

- various visualization techniques adequate to measurement scales,

- chi-square statistics to verify the independence between categorical variables,

- contingency coefficient to evaluate the associations between nominal variables,

- Kendall's tau-b coefficient as well as Goodman and Kruskal gamma coefficient to assess the associations between ordinal variables,

- Quetelet indexes to detect the category-to-category degree of influence (Mirkin, 2001),

- nonlinear canonical correlation analysis to represent the relationship between two sets of variables.

Most visualization techniques are accessible in $\mathrm{R}$ program.:

- matrix visualizations using colour intensity and pie representation (see Friendly, 2002), applied to depict contingency tables, are made by functions available in corrplot package (Tei, 2013),

- barcode plots revealing granularity of data, used to show relationships between age and attitudes given in ordinal scales are done with application of barcode package (Emerson, Green \& Hartigan, 2012),

- conditional density plots (see Everitt \& Hothorn, 2009, pp. 37-38) showing relationships between age and attitudes given in ordinal scales by surface and colour A are derived through vcd package (Meyer, Zeileis \& Hornik, 2013).

Only records containing all data are taken into consideration giving the sample size $\mathrm{N}=1844$.

\section{Raw Data Visualizations and Categorization}

Visualization techniques are useful to gain a general insight into data allowing to reveal certain patterns and regularities. Preliminary visual representation of the raw data showing relations between interpersonal trust components, gender and age are given in Figure 1 and 2.

Figure 1. Visualization of raw contingency tables: gender and 0-10 scale answers to questions representing interpersonal trust components

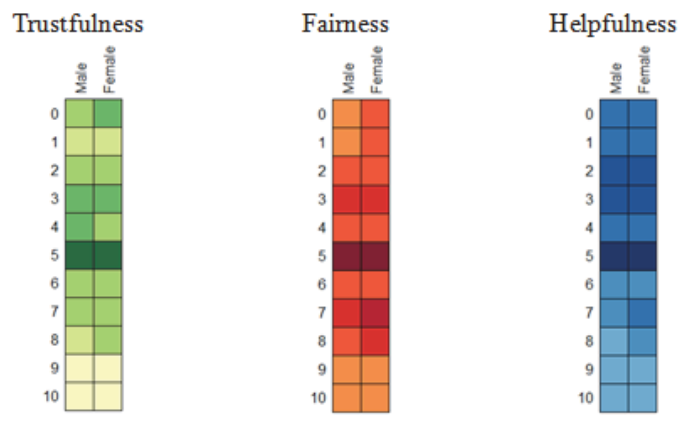

Source: own elaboration with $\mathrm{R}$, corrplot package 
Figure 2. Barcode visualization of relationships between age and 0-10 scale answers to questions representing interpersonal trust components
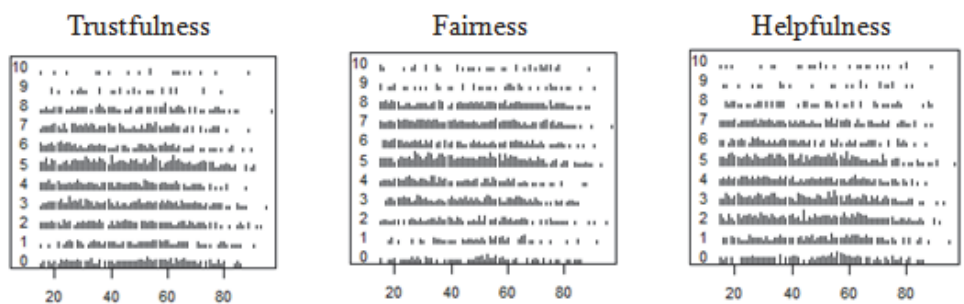

Source: own elaboration with $\mathrm{R}$, barcode package

Figure 1 represents the frequencies in contingency tables cells by the colour intensity. The stripes in Figure 2 represent the occurrences of categories corresponding to certain age. Domination of neutral category (5) can be recognized easily as well as low number of answers corresponding to the high evaluation. As the eleven-range Likert scale was applied it might be reasonable to join some categories for further analysis. The categorization is done as follows:

A answers 0-1, i.e. extremely negative attitude,

$B$ answers 2-4, i.e. negative attitude,

C answer 5, i.e. neutral attitude,

D answers 6-8, i.e. positive attitude,

E answers 9-10, i.e. extremely positive attitude.

Other visualization methods are applied to depict categorized data: matrix vizualizations with pie charts (Friendly, 2002) and conditional density plots (see Everitt \& Hothorn, 2009, pp. 37-38). They are shown on Figure 3 and Figure 4, respectively.

Figure 3. Visualization of contingency tables: gender and categorized answers (A-E) to questions representing interpersonal trust components
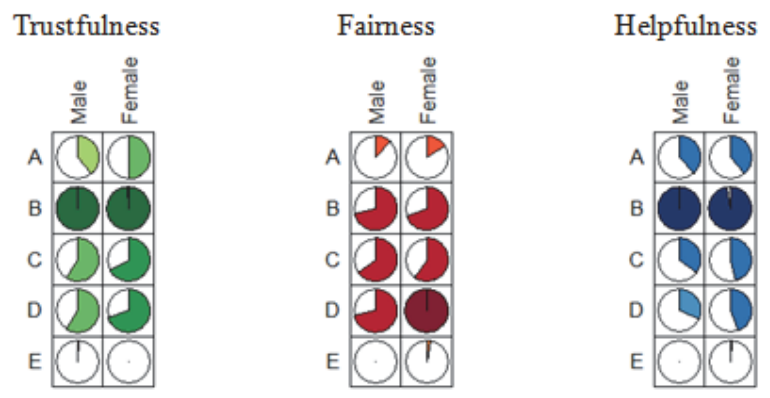

Source: own elaboration with $\mathrm{R}$, corrplot package

Figure 4. Conditional density plot visualization of relationships between age and categorized answers (A-E) to questions representing interpersonal trust components
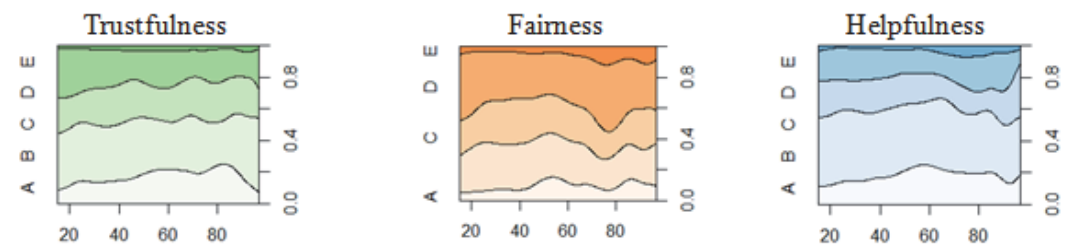

Source: own elaboration with $\mathrm{R}$, vcd package 
Both visualizations reveal that there might be some associations between socio-economic factors as gender or age and attitude to interpersonal trust. Moreover the patterns of association are slightly different for the three considered components. Some quantitative measures are applied in the next paragraph to evaluate the phenomena.

\section{Evaluation of Associations Among Non-Metric Data}

Several methods are applied to assess associations among considered categorical variables. Chi-square statistic is used to verify if there are dependencies between gender or age and responses to questions about interpersonal trust. As chisquare statistic depends on the number of observations it is not suitable to measure the strength of association, so other specific coefficients are calculated, namely contingency coefficients for a combination of nominal and ordinal variables and Kendall's tau-b as well as Goodman and Kruskal gamma coefficients for ordinal variables. The computational formulae of these coefficients are well-known in statistical analysis and can be found for example in Everitt (1992, pp. 57, 62-63). The contingency coefficient lies between 0 and 1, where 0 occurs in case of independence and bigger values suggest a stronger association. Kendall's tau-b and Goodman and Kruskal gamma proposals take into consideration the ordinal character of variables by distinguishing concordant pairs of observations with rankings in the same direction and discordant pairs with rankings in the reverse direction (Everitt, 1992, p. 62). Additionally Kendall's coefficient includes also observations tied to both variables. Both coefficients can take positive and negative values showing the direction of association. They are equal zero in case of independence and their absolute value does not exceed 1.

It is necessary to categorize age to measure the relationships between this variable and other ordinal variables. The categorization is done in such a way that three approximately equinumerous groups are derived, i.e.:

- lower age interval (up to 34 years),

- $\quad$ medium age interval (35-55 years),

- upper age interval (56 years and more).

Chi-square statistic and association coefficients are given in Table 1 and Table 2.

Table 1. Associations between interpersonal trust components and gender

\begin{tabular}{cccc}
\hline Component & $\begin{array}{c}\text { Chi-square } \\
\text { statistic }\end{array}$ & p-value & Contingency coefficient \\
\hline Trustfulness & 7,013 & 0,135 & 0,062 \\
Fairness & 11,658 & 0,020 & 0,079 \\
Helpfulness & 3,791 & 0,435 & 0,045 \\
\hline
\end{tabular}

Source: own computations

Table 2. Associations between interpersonal trust components and age (categorized)

\begin{tabular}{ccccc}
\hline Component & Chi-square statistic & $p$-value & $\begin{array}{c}\text { Kendall's tau-b } \\
\text { coefficient }\end{array}$ & $\begin{array}{c}\text { Gamma } \\
\text { coefficient }\end{array}$ \\
\hline Trustfulness & 27,703 & 0,001 & $-0,057$ & $-0,081$ \\
Fairness & 32,491 & 0,000 & 0,009 & 0,013 \\
Helpfulness & 31,357 & 0,000 & $-0,050$ & $-0,072$ \\
\hline
\end{tabular}

Source: own computations

The chi-square statistics show that in case of gender only one component is significant (fairness), but all components are related with age substantially. However, the degree of association is rather weak. Older Poles tend to evaluate better the fairness than the younger generations. On the contrary, they find people less trustworthy and less helpful. Women are inclined to assess the fairness of others significantly higher than men. It must be kept in mind that the associations among the variables are very poor and perhaps a special measure would reflect better differences in contingency table cells.

Another way to analyze contingency tables was proposed by Quetelet who recommended absolute and relative indexes measuring "local" degree of influence of one category to another by comparing conditional and marginal probabilities (Mirkin, 2001). In this paper a formula representing relative change in probability is applied (Mirkin, 2001): 


$$
q_{i j}=\frac{p(i / j)-p_{i+}}{p_{i+}}
$$

where: $p(\mathrm{i} / \mathrm{j})$ - conditional probability (given that category $j$ occurs), $p_{i+}-$ marginal probability of category $i$.

The values of the indexes are given in Table 3 and in Table 4.

Table 3. Quetelet indexes (\%) reflecting the influence of gender towards categorized answers (A-E) to questions representing interpersonal trust components

\begin{tabular}{ccccccc}
\hline \multirow{2}{*}{ Category } & \multicolumn{2}{c}{ Trustfulness } & \multicolumn{2}{c}{ Fairness } & \multicolumn{2}{c}{ Helpfulness } \\
\cline { 2 - 7 } & Male & Female & Male & Female & Male & Female \\
\hline A & $-6,1$ & 5,6 & $-2,8$ & 2,6 & 3,5 & $-3,2$ \\
B & 4,9 & $-4,5$ & 6,2 & $-5,7$ & 5,9 & $-5,3$ \\
C & $-1,0$ & 0,9 & 9,1 & $-8,3$ & $-6,7$ & 6,1 \\
D & $-3,6$ & 3,2 & $-10,1$ & 9,3 & $-9,3$ & 8,5 \\
E & 13,2 & $-12,0$ & $-7,9$ & 7,2 & $-6,5$ & 5,9 \\
\hline
\end{tabular}

Source: own computations

Table 4. Quetelet indexes (\%) reflecting the influence of age towards categorized answers (A-E) to questions representing interpersonal trust components

\begin{tabular}{cccccccccc}
\hline & \multicolumn{3}{c}{ Trustfulness } & \multicolumn{3}{c}{ Fairness } & \multicolumn{3}{c}{ Helpfulness } \\
\cline { 2 - 9 } Category & \multicolumn{3}{c}{ Age interval } & \multicolumn{3}{c}{ Age interval } & \multicolumn{3}{c}{ Age interval } \\
& lower & medium & upper & lower & medium & upper & lower & medium & upper \\
\hline A & $-25,4$ & $-2,0$ & 27,5 & $-31,7$ & 12,7 & 19,2 & $-27,6$ & 3,9 & 23,9 \\
B & 5,5 & 3,4 & $-9,0$ & 5,9 & 5,9 & $-11,7$ & 7,0 & $-2,2$ & $-4,8$ \\
C & $-5,3$ & 3,7 & 1,6 & $-0,8$ & 10,8 & $-10,0$ & 6,3 & 10,1 & $-16,4$ \\
D & 18,5 & $-10,4$ & $-8,2$ & 9,4 & $-15,0$ & 5,5 & 11,0 & $-7,1$ & $-4,0$ \\
E & $-28,4$ & 26,8 & 1,9 & $-34,4$ & $-10,5$ & 45,0 & $-36,1$ & $-13,8$ & 49,9 \\
\hline
\end{tabular}

Source: own computations

Quetelet indexes reveal various patterns on the influence of gender and age towards three interpersonal trust components. Generally, the indexes are higher in case of age as compared to gender what confirms earlier findings. There are some particular results worth underlying:

- category "female" is positively related to high assessment of fairness and helpfulness (categories D and E),

- category "male" is strongly positively related to very high evaluation of trustfulness (category E),

- category "lower age interval" is negatively related to extreme categories ( $\mathrm{A}$ and $\mathrm{E}$ ) and positively related to more moderate opinions ( $B$ and $D$ ),

- category "upper age interval" is related to more polarized opinions (categories A and E),

- category "medium age interval" is positively related to negative and neutral attitudes to fairness as well as to a very high assessment of trustfulness.

All considered indicators show that there are some differences in perception of the interpersonal trust components with respect to gender and age, but the degree of associations is low.

\section{Combining Things Together - A Multivariate Approach}

In this study two sets of variables are considered. The first one corresponds to opinions about interpersonal trust, the second one comprises such factors as age and gender. Relationships between pairs of variables are discussed in paragraphs 3 and 4 . This part of the study attempts to combine all variables together and carry out the analysis for all of them simultaneously. As the variables are non-metric and constitute two sets of different nature nonlinear canonical correlation analysis (see Meulman \& Heiser, 2001) is chosen to determine and visualize the associations among categories. Two dimensional solution is proposed for the analysis giving a representation on a plane (Figure 5). The first coordinate represents approximately $52 \%$ of captured relationship and the second coordinate about $48 \%$. Unfortunately, the actual fit of the solution is rather poor $(55,6 \%)$ so the results seem not very informative. 
Figure 5. Results of nonlinear canonical correlation analysis

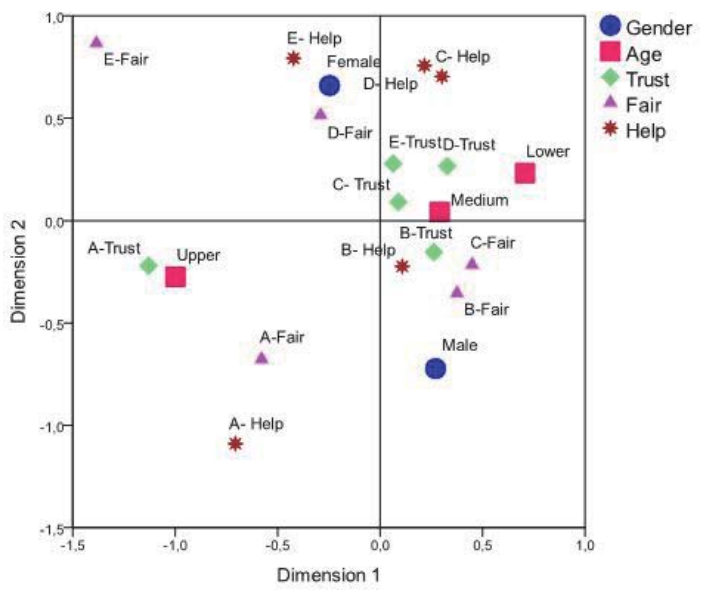

Source: own computations with IBM SPSS Statistics 21.

However, some general conclusions can be drawn. There is a strong contrast between upper age category and other age categories. This is combined with evaluation of trust. The position of points lying in upper left and lower right parts of the plot reflect relationships of gender and to some extent fairness and helpfulness. Older people tend to present extremely negative attitude to interpersonal trust. Women evaluate helpfulness and fairness higher than men.

\section{Conclusions}

Different types of data visualizations give an opportunity to show the overall relations in datasets. Such presentations which are less detailed as tables with particular numbers allow to recognize some underlying patterns immediately. Visualization techniques give a general insight into data patterns and are very useful when analyzing socio-economic data.

Chi-square statistics allow to infer that there are significant relations between age and interpersonal trust components. No such evidence can be found when regarding gender - a significant dependence occurs in case of the element "fairness" only.

Although chi-square test reveals significant dependences, association measures indicate that the strength of them are very weak. There is a positive relationship between age and component "fairness" and negative dependence between age and attitude to trustfulness or helpfulness. The degree of influence of gender and age category on the interpersonal trust elements may be evaluated by Quetelet indexes. According to them the influence is much stronger in case of age than in case of gender

A multivariate approach, i.e. nonlinear canonical correlation analysis gives an interesting opportunity to visualize the associations among all variables taken together and grouped into sets but due to poor fit is rather not reliable in this case.

All results obtained in this study indicate that there is no one universal pattern of the relations between gender or age and three interpersonal trust components. Moreover, even if associations exist they are rather weak.

\section{Acknowledgments}

This research was financed by Narodowe Centrum Nauki (National Science Centre) in Poland under the project entitled "Non-metric multivariate data analysis as a tool for study of adults situation in the context of demographic changes". Project number: 2012/05/B/HS4/02499.

This paper uses European Social Survey data: ESS Round 6: European Social Survey Round 6 Data (2012). Data file edition 1.1, European Social Survey Norwegian Social Science Data Services, Norway - Data Archive and distributor of ESS data. 


\section{References}

Czapiński J. \& Panek T. (eds.), Social diagnosis 2013. Objective and subjective quality of life in Poland. Diagnoza społeczna 2013. Warunki i jakość życia Polaków. (2013) Contemporary Economics, Vol.7, Rada Monitoringu Społecznego, Warszawa.

Emerson J. W., Green W. A., \& Hartigan J.A. (2012). barcode: Barcode distribution plots, R package ver. 1.1, http://cran.rproject.org/web/packages/barcode/index.html

Everitt, B. S. (1992). The analysis of contingency tables (Vol. 45), CRC Press.

Everitt, B. S., \& Hothorn, T. (2009). A handbook of statistical analyses using R. CRC Press.

Friendly, M. (2002). Corrgrams: Exploratory displays for correlation matrices. The American Statistician, 56(4), 316-324.

Grześkowiak A. Assessment of interpersonal trust of Poles by principal components analysis and log-linear modeling, Ekonometria 2014 (forthcoming)

Meulman, J. J., \& Heiser, W. J.(2001). SPSS Categories 11.0. SPSS, Chicago.

Meyer D., Zeileis A., \& Hornik K. (2013). vcd: Visualizing Categorical Data. R package version 1.3-1, http://cran.rproject.org/web/packages/vcd/index.html

Mirkin, B. (2001). Eleven ways to look at the chi-squared coefficient for contingency tables. The American Statistician, 55(2), 111-120.

Morrone, A., Tontoranelli, N., \& Ranuzzi, G. (2009). How Good is Trust?: Measuring Trust and its Role for the Progress of Societies OECD Statistics Working Papers, 2009/3, OECD Publishing

Wei T. (2013). corrplot: Visualization of a correlation matrix, R package ver. 0.73 , http://cran.r-project.org/web/packages /corrplot/index.html

Zaufanie społeczne. Komunikat z badań (2012), CBOS, Warszawa. http://www.cbos.pl/ SPISKOM.POL/2012/K_033_12.PDFretrieved ) .(14-01-2014 